\title{
Phylogenetic community structure in Minnesota oak savanna is influenced by spatial extent and environmental variation
}

\author{
Charles G. Willis, Marta Halina, Clarence Lehman, Peter B. Reich, Adrienne Keen, \\ Shawn McCarthy and Jeannine Cavender-Bares
}

C. G. Willis, M. Halina, C. Lehman, A. Keen, S. McCarthy and J. Cavender-Bares (cavender@umn.edu), Dept of Ecology, Evolution and Behavior, 1987 Upper Buford Circle, Univ. of Minnesota, St. Paul, MN 55108, USA. (Present address of C. G. W.: Dept of Biology, 125 Science Drive, Duke Univ., Box 90338, Durham, NC 27708, USA, present address of M. H.: Dept of Philosophy, Univ. of California, San Diego, 9500 Gilman Drive, La Jolla, CA 92093, USA, present address of A. K.: London School of Hygiene and Tropical Medicine, 49 Bedford Square, London, WC1B3DP, UK.) - P. B. Reich, Dept of Forest Resources, 220f Green Hall, Univ. of Minnesota, St. Paul, MN 55108, USA.

\begin{abstract}
The relative importance of environmental filtering, biotic interactions and neutral processes in community assembly remains an openly debated question and one that is increasingly addressed using phylogenetic approaches. Closely related species may occur together more frequently than expected (phylogenetic clustering) if environmental filtering operates on traits with significant phylogenetic signal. Recent studies show that phylogenetic clustering tends to increase with spatial scale, presumably because greater environmental variation is encompassed at larger spatial scales, providing opportunities for species to sort across environmental gradients. However, if environmental filtering is the cause of species sorting along environmental gradients, then environmental variation rather than spatial scale per se should drive the processes governing community assembly. Using species abundance and light availability data from a long-term experiment in Minnesota oak savanna understory communities, we explicitly test the hypothesis that greater environmental variation results in greater phylogenetic clustering when spatial scale is held constant.

Concordant with previous studies, we found that phylogenetic community structure varied with spatial extent. At the landscape scale $(\sim 1000 \mathrm{ha})$, communities were phylogenetically clustered. At the local scale $(0.375 \mathrm{ha})$, phylogenetic community structure varied among plots. As hypothesized, plots encompassing the greatest environmental variation in light availability exhibited the strongest phylogenetic clustering. We also found strong correlations between species functional traits, particularly specific leaf area (SLA) and perimeter per area (PA), and species light availability niche. There was also a phylogenetic signal in both functional traits and species light availability niche, providing a mechanistic explanation for phylogenetic clustering in relation to light availability. We conclude that the pattern of increased phylogenetic clustering with increased environmental variation is a consequence of environmental filtering acting on phylogenetically conserved functional traits. These results indicate that the importance of environmental filtering in community assembly depends not on spatial scale per se, but on the steepness of the environmental gradient.
\end{abstract}

The composition of local communities is thought to depend on the availability of species (i.e. the regional species pool), species ability to reach a given locale (i.e. dispersal), and whether species can persist given the local abiotic and biotic environment (e.g. environmental filtering and species interactions). The debate over the relative importance of these mechanisms in structuring communities is long standing in community ecology (Diamond 1975, Hubbell 1979, 2001, Tilman 1982, Ricklefs 1987, Chesson 1991, Ricklefs and Schluter 1993, Bazzaz 1996, Weiher and Keddy 1999). Currently, much debate centers on the relative importance of neutral processes (e.g. historical processes that influence the species pool, stochasticity, and dispersal) versus deterministic processes (e.g. environmental filtering, competitive exclusion, and other density dependent interactions) (Hubbell 2001, Chase and Leibold 2003, Fargione et al.
2003, Tilman 2004, Leibold and McPeek 2006). There is abundant evidence that niche-based processes are important in structuring communities (Parrish and Bazzaz 1976, Tilman 1982, Weiher et al. 1998, Silvertown et al. 1999, Chase and Leibold 2003, Reich et al. 2003, Ackerly 2004a, Cavender-Bares et al. 2004a, Dybzinski and Tilman 2007, Kraft et al. 2008). However, the relative importance of niche-based and neutral processes remains unclear, in part because processes are not mutually exclusive and may shift in importance at different scales or in different contexts (Allen and Starr 1982, Weiher and Keddy 1999, Tilman 2004, Kembel and Hubbell 2006, Leibold and McPeek 2006). For instance, the importance of environmental filtering is hypothesized to be dependent on the steepness of the environmental gradient, i.e. the amount of environmental variation, within a community. The steeper the gradient, the 
more likely species will sort according to their relevant functional traits (Bazzaz 1996, Fig. 1).

Recently, community ecologists have taken advantage of the evolutionary relationships of species (i.e. phylogenetics) to help infer predominant processes underling community structure (Webb 2000, Webb et al. 2002, Ackerly 2004b, Cavender-Bares et al. 2004b, 2009, Kembel and Hubbell 2006, reviewed in Slingsby and Verboom 2006, Emerson and Gillespie 2008, Vamosi et al. 2009). Species evolutionary history influences both the regional species pool and species functional traits (Webb et al. 2002, Wiens and Donoghue 2004, Hardy and Senterre 2007). With regard to species functional traits, biologists since Darwin have argued that closely related species share similar functional traits due to shared ancestry (i.e. trait conservatism) and thus are ecologically similar (i.e. niche conservatism) (Darwin 1859, Wiens and Graham 2005, Donoghue 2008). Testing the assumption of niche conservatism is important for inferring community assembly processes from phylogenetic patterns (Losos 2008). If environmental filtering is the predominant assembly process, then closely related species are more likely to co-occur (i.e. phylogenetic clustering) if they share niche preferences.

An important pattern to emerge from recent studies of phylogenetic community structure is that phylogenetic clustering increases with spatial extent (Cavender-Bares et al. 2006, Slingsby and Verboom 2006, Swenson et al. 2007). This pattern is hypothesized to be caused by the increased importance of environmental filtering resulting from greater environmental variation encompassed at broader spatial extents.

However, several factors confound the assumption that greater phylogenetic clustering at larger spatial extent is the result of environmental filtering. First, trait conservatism cannot be assumed, nor can it be assumed to be equivalent to niche conservatism (Losos 2008). Moreover, closely related species may be ecologically divergent following an adaptive radiation (Schluter 2000, Cavender-Bares et al. $2004 \mathrm{~b}$ ), species might have adapted to the same niche with different traits, particularly in key leaf functional traits (Wainwright et al. 2005), or conserved traits that are statistically associated with patterns of phylogenetic clustering may not be functionally linked to species niches.

Addressing these caveats requires assessing the phylogenetic signal and understanding the functional importance of species traits in a given system. A growing literature has demonstrated broad patterns of phylogenetic conservatism in plant traits (Ackerly and Reich 1999, Prinzing et al. 2001, Ackerly 2004b, Cavender-Bares et al. 2006, Kerkhoff et al. 2006). These functional traits are known to be generally important in determining global and local species distributions with regard to water availability, temperature, and light availability (Kitajima 1994, Reich et al. 1999, Wright et al. 2001, Reich et al. 2003, Ackerly 2004a, Lusk and Warton 2007). Such studies provide grounds for making a priori predictions on what traits might be

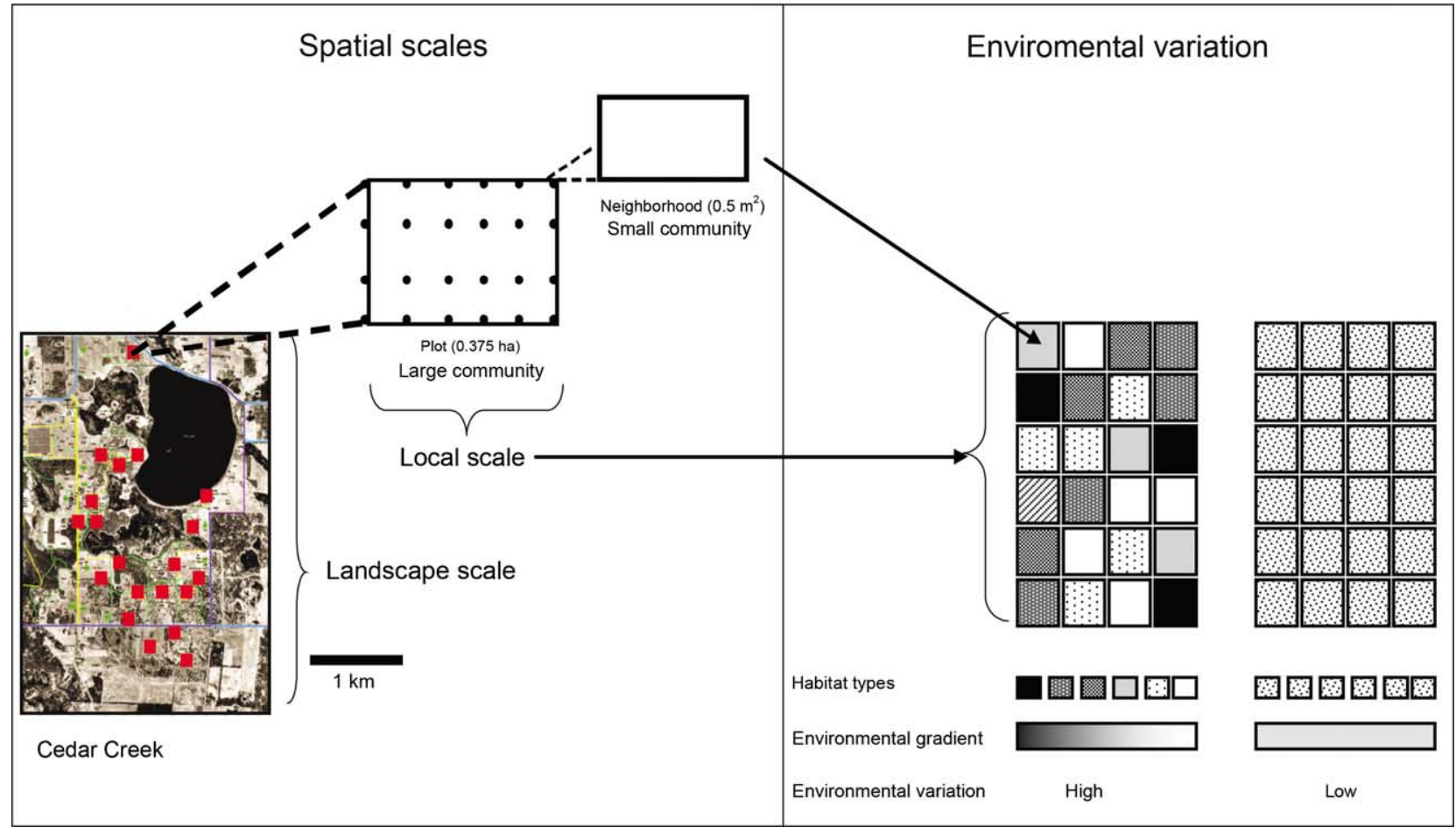

Figure 1. A graphical explanation of the spatial scales and environmental variation of the long-term ecological oak savanna fire experiment Cedar Creek Natural History Area (CCNHA), Minnesota. Spatial scales include both the size of the community being compared (small vs large) and the spatial extent (local vs landscape) across which communities were compared (left panel). Environmental variation can occur across plots and across neighborhoods within plots (shown as squares within plots in the right panel). Greater environmental variation within plots results in a steeper environmental gradient across which species are distributed. If the distribution of species across this environmental gradient is determined by evolutionarily conserved functional traits, then a pattern of increased phylogenetic clustering with increased environmental variation is predicted. 
important in structuring a particular community. However, it is unclear to what extent these a priori predictions will hold when tested for in a given community, particularly at the local scale. Linking traits chosen a priori to phylogenetic clusteringin novel communities requires validation that the traits have phylogenetic signal and functional importance in the system under study.

A second issue also complicates the assumption that greater phylogenetic structuring at larger spatial scales is the result of environmental filtering. Studies that have observed increasing phylogenetic clustering with spatial extent (Cavender-Bares et al. 2006, Slingsby and Verboom 2006, Swenson et al. 2007) encompass relatively large geographic regions, permitting the possibility that the geography of speciation could contribute to the pattern of phylogenetic clustering at the regional scale. For example, if speciation occurred in situ with limited dispersal, assemblages of closely related species might reflect biogeographic processes of speciation rather than ecological processes (Ricklefs and Schluter 1993, Losos and Glor 2003, Ricklefs et al. 2004, Wiens and Donoghue 2004, Kozak et al. 2005). Alternatively, it is possible that environmental gradients that are most characteristic at broad spatial extent, such as climate, could have a greater environmental filtering effect than environmental gradients that operate locally within communities or ecosystems (e.g. light). This could also lead to greater phylogenetic clustering with spatial extent due to the interplay of spatial scale and turnover in environmental gradients.

Finally, as noted above, for some environmental factors variation and spatial extent are likely to co-vary, making it statistically difficult to tease the two apart. Thus, to test the effect of environmental variation on phylogenetic community structure it is necessary to account for these potential caveats by: 1) accounting for the historical biogeography of the species pool, 2) testing for phylogenetic signal in traits determined to be functionally relevant or in the environmental niche itself, and 3) holding spatial extent constant while varying variation of specific environmental factors within communities.

Here, we take advantage of a long-term fire-frequency experiment in an oak savanna-woodland ecosystem (hereafter oak savanna) at Cedar Creek Ecosystem Science Reserve (formerly Cedar Creek Natural History Area) in Minnesota, USA to examine the relationship between phylogenetic community structure, spatial extent, and environmental variation. This system consists of twentynine 0.375 ha plots composed of twenty-four evenly distributed $0.5 \mathrm{~m}^{2}$ quadrants with detailed species abundance and light availability data (Fig. 1). These plots all occur in a $\sim 1000$ ha reserve, and are thus exposed to the same regional species pool, eliminating the potential for historical biogeographic effects. The availability of multiple plots allows us to compare phylogenetic community structure across communities that vary in environmental variation, while holding spatial extent constant.

We first test for emergent patterns of phylogenetic clustering as evidence of environmental filtering at two different spatial extents (i.e. local scale $[0.375 \mathrm{ha}]$ vs landscape scale $[\sim 1000$ ha], Fig. 1$)$. Second, we test a set of species traits for their functional importance in determining species distribution across a light gradient. Third, we test whether these same functional traits and species light availability niches are conserved and can thus explain any patterns of phylogenetic clustering we observe. Finally, we specifically test the hypothesis that phylogenetic clustering increases with environmental variation by testing if phylogenetic community structure within plots correlates with environmental variation within plots, holding spatial extent constant.

\section{Methods}

\section{Site history, environmental gradients, and data collection}

Cedar Creek Ecosystem Science Reserve (referred to hereafter as Cedar Creek) is located in central-eastern Minnesota $\left(45^{\circ} 25^{\prime} \mathrm{N}, 93^{\circ} 10^{\prime} \mathrm{W}\right)$ on the Anoka Sand Plain, a glacial washout of relatively flat, low nutrient, sandy soil. Cedar Creek lies near the intersection of three major North American biomes: northern pine forest, western tall-grass prairie, and eastern deciduous forest. The latter two biomes intermingle at Cedar Creek to create the ecologically distinct ecotone of temperate oak savanna.

Minnesota temperate oak savanna is characterized by a mixture of tall-grass prairie grasses and forbs interspersed with two dominant oak species, burr oak Quercus macrocarpa and northern pin oak Q. ellipsoidalis, as well as other northern woody species. The dynamic equilibrium between prairie and forest that results in temperate oak savanna has historically been maintained by frequent fires which repressed tree sapling and shrub-growth and maintained canopy openings (Peterson and Reich 2008). After European settlement, the suppression of fire, along with agricultural clearing, led to significant collapse of the traditional open-canopy oak savanna vegetative community, which disappeared or was converted to closed canopy deciduous oak woodland with dense underbrush (Peterson and Reich 2008).

In 1964, an experiment with differing frequencies of prescribed burns was begun at Cedar Creek on savanna areas that had never been cleared for agriculture. Since 1964, the project has expanded to twenty-nine burn units (ranging from 3 to 27 ha in area), with fire frequencies ranging from fires in 4 of $5 \mathrm{yr}$ to no burns (for more details see Peterson and Reich 2008). Within these burn units, twenty-nine permanent $50 \times 75 \mathrm{~m}$ (0.375 ha) study plots have been established with typically one plot per burn unit. Within each plot are twenty-four regularly spaced $1 \times$ $0.5 \mathrm{~m}\left(0.5 \mathrm{~m}^{2}\right)$ quadrats, referred to herein as neighborhoods (Fig. 1). As a result of prior vegetation conditions and fire history, large gradients in light availability exist both within and among burn units and study plots (Peterson and Reich 2008). Although fire frequency and light availability are significantly correlated, the correlation explains a minor fraction of total variation, and light availability has been shown to be a dominant driver of community composition (Peterson and Reich 2008).

Understory species percent cover was measured from breast height in each $0.5 \mathrm{~m}^{2}$ neighborhood and used to estimate species abundance, as previously described (Peterson and Reich 2008). Plots were surveyed during the summer of 
2005. Within neighborhoods, the number of species ranged from 1 to 24 with a median number of 10 . Patterns of phylogenetic community structure were tested using the abundance data from these neighborhood surveys as described in the sections that follow. Neighborhoods were oriented to avoid the inclusion of plants growing above $\mathrm{BH}$ (e.g. trees, shrubs) and large detritus that would reduce the effective size of the neighborhood (e.g. logs, thatch ant mounds). When plants growing above $\mathrm{BH}$ or large detritus did occur in a neighborhood, they were scored as "litter/ other" and were not included in the analysis. Only 23 of 696 neighborhoods contained "litter/other" that covered $>20 \%$ of the area. When these neighborhoods were completely removed from our analysis, our results were highly similar to those reported below (data not shown).

\section{Environmental data}

Light availability within each neighborhood was determined by the amount of overstory canopy cover and was measured as diffuse transmittance ratio (DIFN), where 0 represents a fully closed canopy and 1 represents open sky; the measure correlates closely with incident photo flux irradiance (Machado and Reich 1999). Measurements were taken in either the late morning or early evening hours using a LAI2000 sensor during the summer of 2005 (Reich unpubl.). Light measurements were taken just above the censused understory vegetation. Therefore, species in our community data did not contribute to the light environment of the neighborhood in which they occurred.

\section{Trait data}

Species functional traits were obtained from both field collections and available literature. Traits were chosen based on a priori predictions of their functional importance in relation to light availability. The functional relevance of each trait is justified below.

Specific leaf area $\left(\mathrm{cm}^{2} \mathrm{~g}^{-1}\right)$ [SLA] is predicted to be negatively correlated with increasing light availability. Higher SLA in low light maximizes light interception and net $\mathrm{C}$ gain per unit investment in $\mathrm{C}$ and $\mathrm{N}$, whereas lower SLA in high light improves hydraulic conductance and protects against photoinhibition and desiccation (Givnish and Vermeij 1976). Specific leaf area is also expected to vary inversely with light availability as part of the closely coordinated suite of traits (including leaf nitrogen, photosynthetic rate, and respiration rate) whose variation allows ecological success in any given community of species with differing trait syndromes because of the trade-offs along the leaf economic spectrum that occur in relation to resource supply variation (Wright et al. 2004).

The expected correlation between SLA and light availability has been demonstrated empirically in previous studies on deciduous perennials with relatively short leaflife spans. In a meta-analysis, Lusk and Warton (2007) found a positive correlation between SLA and shade tolerance (an inverse proxy for light availability niche) within deciduous saplings. Similarly, other studies have found negative correlations between SLA and irradiance (Kitajima 1994, Ackerly 2004a).

Average plant height at maturity $(\mathrm{m})$ is predicted to increase with decreasing light availability at breast height, as greater maximum plant height increases competitiveness for access to light under denser canopies (King 1990). Mean individual leaf area per leaf $\left(\mathrm{cm}^{2}\right)$ and leaf perimeter per area $\left(\mathrm{cm}^{-1}\right)[\mathrm{PA}]$ are predicted to decrease with higher light availability because they provide hydraulic and thermal advantages; also, less surface area is needed to capture light for photosynthesis (Sack et al. 2003). Minimum rooting depth $(\mathrm{m})$ was predicted to be positively correlated with increasing light availability as a means of increasing access to water resources as well as increasing ability to recover after fire (Menges and Kohfeldt 1995, Kozlowski and Pallardy 2002, Ackerly 2004a), given that fire frequency and light availability are correlated (Peterson and Reich 2008).

Leaf traits were measured from the field on a subset of 114 species out of the total 192 unique species identified from the abundance data. The youngest fully expanded leaves were collected from 4 to 6 individuals of each species from within or near the experimental plots between June and August 2005. Individuals were chosen opportunistically along random walks within experimental plots. No more than two individuals per species were taken from a given plot.

Phenotypic plasticity can result in intraspecific trait variation and can mirror interspecific trait variation along an environmental gradient. For instance, an alternative dataset collected from the same Cedar Creek plots found 27 of 34 species to have significant negative correlations between SLA and light availability (data not shown). To control for the effects of intraspecific plasticity, leaves were taken only from sunlit locations except in cases where species did not occur in direct sunlight. Furthermore, a test of the impact of plasticity on SLA was run with the dataset of 34 species collected from the same plots, in which SLA of multiple individuals of each species was assessed across a light availability gradient. Specific leaf area was adjusted by light availability as least-square means in an analysis of covariance. This adjustment, however, did not affect the correlation between SLA and light availability $(r=-0.49$, $\mathrm{p}=0.0041$ ). Thus, phenotypic plasticity in SLA is not likely to have a major role in explaining the relative distribution of species across the gradient.

After collection, leaves were placed in distilled water for a minimum of $\sim 2 \mathrm{~h}$ to limit the effect of desiccation. Leaves were then scanned to measure leaf area and perimeter using the software ImageJ (Rasband 19972006). After scanning, leaves were dried at $\sim 60^{\circ} \mathrm{C}$ for a minimum of $48 \mathrm{~h}$ before being weighed for dry leaf weight. Measurements were averaged by species. Measurements were restricted to the primary light capturing area of the leaf; this excluded the petiole or sheath in grasses.

Average plant height at maturity and minimum soil depth required for persistence (minimum root depth) were obtained from the U.S. Dept of Agriculture PLANTS Database (USDA NRCS PLANTS Database 2006). 


\section{Trait-environment correlations}

To test their functional importance, species traits were tested to see if they correlated with species occurrence along the light gradient (i.e. species light availability niche). Species light availability niches were estimated as species mean light availability values $\left(u^{*}\right)$ weighted by their abundance:

$\mathrm{u}^{*}=\sum_{\mathrm{i}} \mathrm{x}_{\mathrm{ij}} \mathrm{y}_{\mathrm{j}}$,

where $x_{i j}$ is the relative abundance of species $i$ in neighborhood $j$, and $y_{j}$ is the environmental value for neighborhood j. Values were summed over all neighborhoods in all plots.

Univariate regression analysis was used to calculate the relationship between each trait and species $\mathrm{u}^{*}$ using the $\mathrm{R}$ function "glm" (R v. 2.6.1, <www.r-project.org/>). Multivariate regression analysis was also performed on all traits with species $\mathrm{u}^{*}$. All traits were $\log$ transformed to satisfy assumptions of normality.

Standard trait-environment correlations, however, can be biased by species shared evolution (Felsenstein 1985). To account for evolutionary history in trait-environmental correlations, we used a modified generalized estimating equation (GEE, Paradis and Claude 2002) implemented in the $\mathrm{R}$ package APE (Paradis et al. 2004). GEE is similar to a generalized linear equation, in that they can perform univariate and multivariate regression analysis. GEE models, however, incorporate a phylogenetic distance matrix that accounts for species relatedness. We performed a regression analysis between each trait with species $\mathrm{u}^{*}$, as well as a multivariate regression analysis of all traits with species $u^{*}$. To correct for multiple tests a sequential Bonferroni correction was performed using the number of traits measured $(\mathrm{k}=5)$.

\section{Phylogeny construction and branch length estimation}

A phylogenetic tree was constructed for 261 vascular plant (Tracheophytes) species recorded in the plots. Initial phylogenetic structure was determined using Phylomatic (Webb and Donoghue 2005). Phylogenetic trees were further resolved based on the most current literature available (references in Supplementary material Appendix 1) using the tree-editing software Mesquite v 2.6 (Maddison and Maddison 2006). In resolving phylogenetic relationships, preference was given to current molecular phylogenies, particularly those using Maximum Likelihood or Bayesian estimation. Relationships that could not be satisfactorily resolved were left as polytomies. The tree is included as a Nexus file in Supplementary material Appendix 1.

Branch length distances were estimated based on minimum node ages (Supplementary material Appendix 2) from the fossil record, based on Wikström et al. (2001). Branch lengths were interpolated from dated nodes using the branch length adjustment (bladj) algorithm in Phylocom v. 4.0.1 (Webb et al. 2008). While there are known limitations to the reliability of branch lengths estimated using this approach (Webb et al. 2008), it represents an important advance over methods that use nodal distances as proxies for branch lengths. It also takes advantage of existing phylogenetic data, synthesizing published expert knowledge, without the considerable expense and time involved in developing molecular phylogenies. We used sensitivity analyses (described below) to determine the robustness of the results to phylogenetic uncertainty.

\section{Phylogenetic uncertainty and sensitivity analyses}

To determine how robust the results of the analyses were to uncertainties in the supertree construction, we tested the sensitivity of our results to variations in tree sensitivity (i.e. topology) and node ages. To test the effects of topology on community phylogenetic analysis, 25 randomized trees were created, where all polytomies were randomly resolved using the program Mesquite v. 2.6. For trait analysis, a separate set of 50 trees were created. All trees are available in Newick format upon request. Analyzes were re-run on each given set of trees. The means and standard errors of each statistic were compared to our observed values to see if they were notably different.

To test branch length sensitivity, we preformed all analyzes using a phylogeny with branch lengths adjusted for divergence time estimates based on Wikström et al. (2001) [Supplementary material Appendix 2], and a phylogeny with branch lengths set to 1 .

\section{Phylogenetic signal of functional traits and species niche}

Phylogenetic signal was determined for species traits and light availability niche. Traits and light availability niche were treated as continuous. Traits were $\log$-transformed when necessary to meet the assumption of normality. Phylogenetic signal was calculated using the "Phylosignal" module in the R-based package "Picante" (Kembel et al. 2008). The module tests the average magnitude of the variances of the descendent trait means across all nodes weighted by branch lengths (Webb et al. 2008). The observed mean variance is then compared to a null distribution generated by randomly shuffling trait values across the tips of the tree 9999 times. If the observed mean variance of the trait ranks $\leq 250$ relative to the null distribution, it is considered to have a significant signal of phylogenetic conservatism (i.e. groups of closely related species tend to have similar traits).

\section{Phylogenetic community structure}

Phylogenetic community structure was analyzed by correlating the phylogenetic distance between species pairs with their degree of co-occurrence (Cavender-Bares et al. 2004b). Phylogenetic distance was estimated using branch lengths adjusted for known node ages. Species pairwise co-occurrence values $\left(\mathrm{C}_{\mathrm{ih}}\right)$ were calculated based on proportional similarity (Schoener 1970):

$\mathrm{C}_{\mathrm{ih}}=1-0.5^{*} \sum\left|\mathrm{p}_{\mathrm{ij}}-\mathrm{p}_{\mathrm{hj}}\right|$,

where $\mathrm{C}$ is the co-occurrence of species $\mathrm{i}$ and $\mathrm{h}$, and $\mathrm{p}_{\mathrm{ij}}$ is the proportion of percent cover of the ith species in the $j$ th 
plot. Phylogenetic community structure in this study thus refers to the pattern of phylogenetic distance between pairs of species and the extent to which they co-occur within sample units (e.g. neighborhoods $\left[0.5 \mathrm{~m}^{2}\right]$ within a plot or in plots $[0.375 \mathrm{ha}]$ within the landscape). Communities are considered to be phylogenetically clustered when species within units tend to be more closely related to each other than to the species in other units relative to a null model in which species distributions are randomized across units. Phylogenetic overdispersion (or "evenness") is defined as when species within units tend to be more distantly related to each other than to species in other units relative to a null model.

Least mean squares (LMS) correlation and quantile regression were used to test the relationship between cooccurrence and phylogenetic distance of species pairs. Least mean squares correlation-regression analysis is generally used in Mantel-type tests, but it assumes constant variance of the response variable with values of the controlling factor. This assumption may be violated for species co-occurrence data across a range of phylogenetic distances. Quantile regression analysis does not require constant variance and has the advantage of permitting the analysis to focus on the upper bound of the data, reducing the bias of zero cooccurrence between many species pairs (Slingsby and Verboom 2006). For quantile regression, we used the 50th quantile (median), which is most similar to the LMS correlation, and the 75th quantile which is often used for the upper bound (Scharf et al. 1998). In both LMS and quantile regression, a negative correlation would suggest phylogenetic clustering, while a positive correlation would suggest phylogenetic overdispersion. Analysis of phylogenetic community structure at the local scale was restricted to LMS and the 75th quantile given the number of plots (29) and the similarity between LMS and the 50th quantile.

We tested for significance by comparing the observed LMS correlation co-efficient or quantile regression slope to a null model (see next section). Observed LMS correlation coefficients or quantile regression slopes were ranked against a null distribution generated by 999 random iterations of species occurrence across units. The observed rank was than subtracted from 1000 to invert the scale. Significance was determined using a two-tailed test. If the observed rank was $\geq 975$ the community was considered significantly phylogenetically clustered. If the observed rank was $\leq 25$ the community was considered significantly phylogenetically overdispersed. Analyses were performed by a unified collection of programs called EcoPhyl, written in C (CavenderBares and Lehman 2007), based on earlier versions in Visual Basic (Cavender-Bares et al. 2004b, 2006).

\section{Null model}

In the null model, species were permuted 999 times across either neighborhoods or plots, keeping row totals (species abundance) but not column totals (community abundance) constant. This approach permits the retention of highresolution abundance data, although the total biomass within a given community is not constrained. To limit the complexity of the analyses, alternative null models in which either presence/absence of species were permuted across plots keeping row and column totals constant, or in which the tips of the phylogenies were permuted, were not tested. Two previous studies indicate that the results using these three null models are qualitatively very similar (Cavender-Bares et al. 2004b, 2006). Using simulations of the data (Hardy and Senterre 2007), no bias was found in the null model due to the phylogenetic distribution of species abundances that would cause a higher Type I error rate than expected (Hardy 2008).

\section{Assumptions of the analyses}

The above analyses make several standard, but important, assumptions. First, traits were analyzed at the species level using mean values. Tests for phylogenetic signal in traits and trait-environment correlations assume that intra-specific variation is less than inter-specific variation. Given the phylogenetic scale at which species are compared (i.e. across all vascular plants) this is a reasonable assumption and has been demonstrated empirically both here (Methods: Trait data) and elsewhere (Reich et al. 1999). Second, the null models that use randomized species abundances assume that dispersal limitation is minimal at the spatial scale at which the test is conducted (i.e. species can disperse to all plots within several generations) and environmental factors are not spatially structured. In particular, violations of the second assumption could complicate the interpretation of the importance of environmental factors in community assembly and patterns of community structure (Legendre et al. 2002). We found that differences in mean light availability between plots were not correlated with the Euclidean spatial distance between plots $(n=29$, mantel $\mathrm{r}=0.08, \mathrm{p}=0.1194)$, indicating environmental factors are not spatially structured at the landscape scale. Since a species light availability niche was calculated from its occurrences across all plots, spatial structuring of the environment within plots is not likely to bias the calculation, if plots themselves are not spatially structured.

\section{Spatial scaling}

Species pairwise co-occurrence values were calculated over two different spatial extents: across all plots $(\sim 1000 \mathrm{ha}$, landscape scale) and within each plot across all 24 neighborhoods ( 0.375 ha, local scale). For analysis of phylogenetic community structure at the landscape scale, species-abundances were summed among all twenty-four neighborhoods within each plot, and these summed abundances were randomized across all plots. For analysis at the local scale, species-abundances were randomized across all neighborhoods within each plot.

\section{Environmental variation and community structure}

The correlation between the phylogenetic community structure within plots (local scale) and the within-plot environmental variation was used to test for a relationship between environmental variation and phylogenetic community structure, independently of spatial extent. Environmental variation was calculated as the variance $\left(\sigma^{2}\right)$ in light 
availability (i.e. DIFN) of all twenty-four neighborhoods within a plot. The correlation between phylogenetic community structure and environmental variance was calculated with the Pearson's correlation co-efficient and Kendall's tau. The correlation was tested with phylogenetic community structure calculated using both LMS correlation and quantile regression analysis (75th quantiles).

\section{Results}

\section{Environmental sorting of conserved traits}

Univariate regression analysis revealed significant correlations between light availability niche and leaf area, height at maturity, PA and SLA (Table 1). When correcting for phylogenetic relatedness we found light availability niche to be significantly correlated with a slightly different set of traits: height at maturity, PA, rooting depth, and SLA (Table 1).

When all five traits were included in a multivariate model, only the association between SLA and species light availability niche was marginally significant. Similarly, when all five traits where included in multivariate GEE model, only the correlation between SLA and light availability niche remained marginally significant (Table 1 ). However, several of the traits were correlated with one another, in particularly, leaf area and PA (non-phylogenetic $\mathrm{R}=-0.70, \mathrm{n}=39$ ). When leaf area was removed from both the non-phylogenetic and phylogenetic model to reduce the effects of collinearity, PA became significantly negatively correlated with light availability niche (Table 1 for phylogenetic results, results of non-phylogenetic analysis not shown). Additional combinations of traits did not produce notably different results. The reduced data set used in the multivariate model did not affect standard errors of the trait estimates nor did the reduced data set affect the correlation between individual traits and light availability niche (data not shown). When we found significant correlations between traits and the species niche, they were in accordance with our a priori predictions (Methods: Trait data). Phylogenetic trait-environment correlations were not notably sensitive to topological variation, but were sensitive to branch length (Supplementary material Appendix 3). However, the correlation between SLA and light availability niche was insensitive to variation in topology or branch lengths.

Furthermore, significant phylogenetic signal was found for SLA, height at maturity, leaf area, PA, and rooting depth (Table 1). No trait was found to be significantly convergent. Species light availability niche was also found to show significant phylogenetic signal (Table 1, Fig. 2). The phylogenetic signal of individual traits varied somewhat with topology and branch lengths, but the phylogenetic signal of species light availability niche was consistent across analyzes (Supplementary material Appendix 3).

\section{Phylogenetic community structure across spatial scales}

Species pairwise co-occurrence values were more negatively correlated with species pairwise phylogenetic distances than

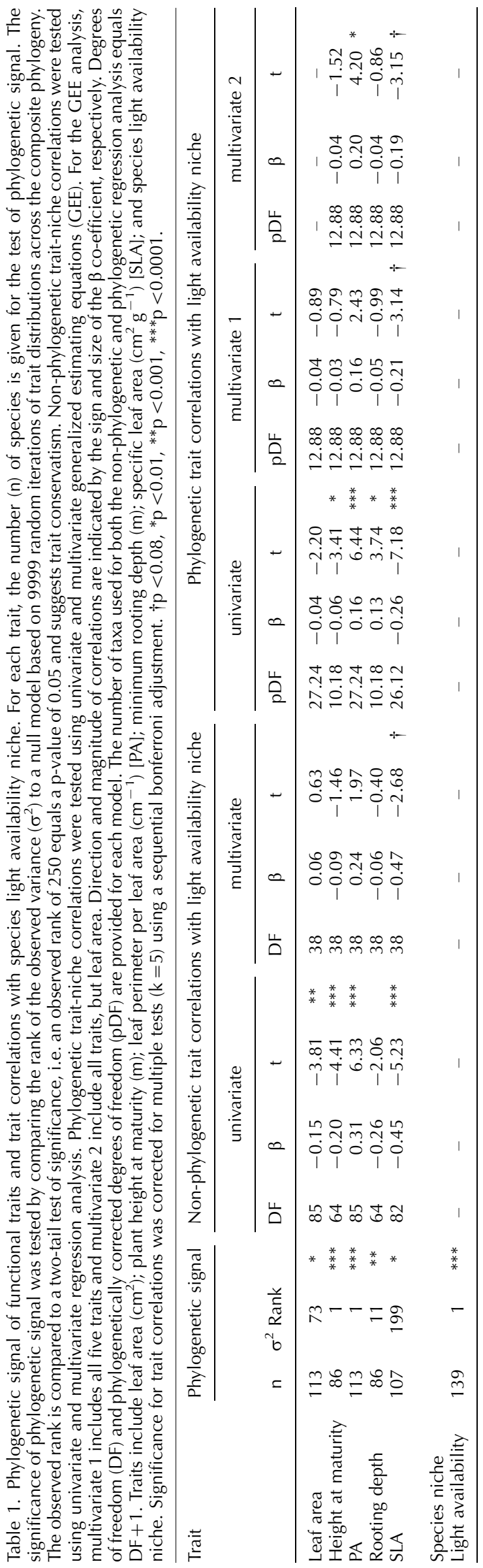




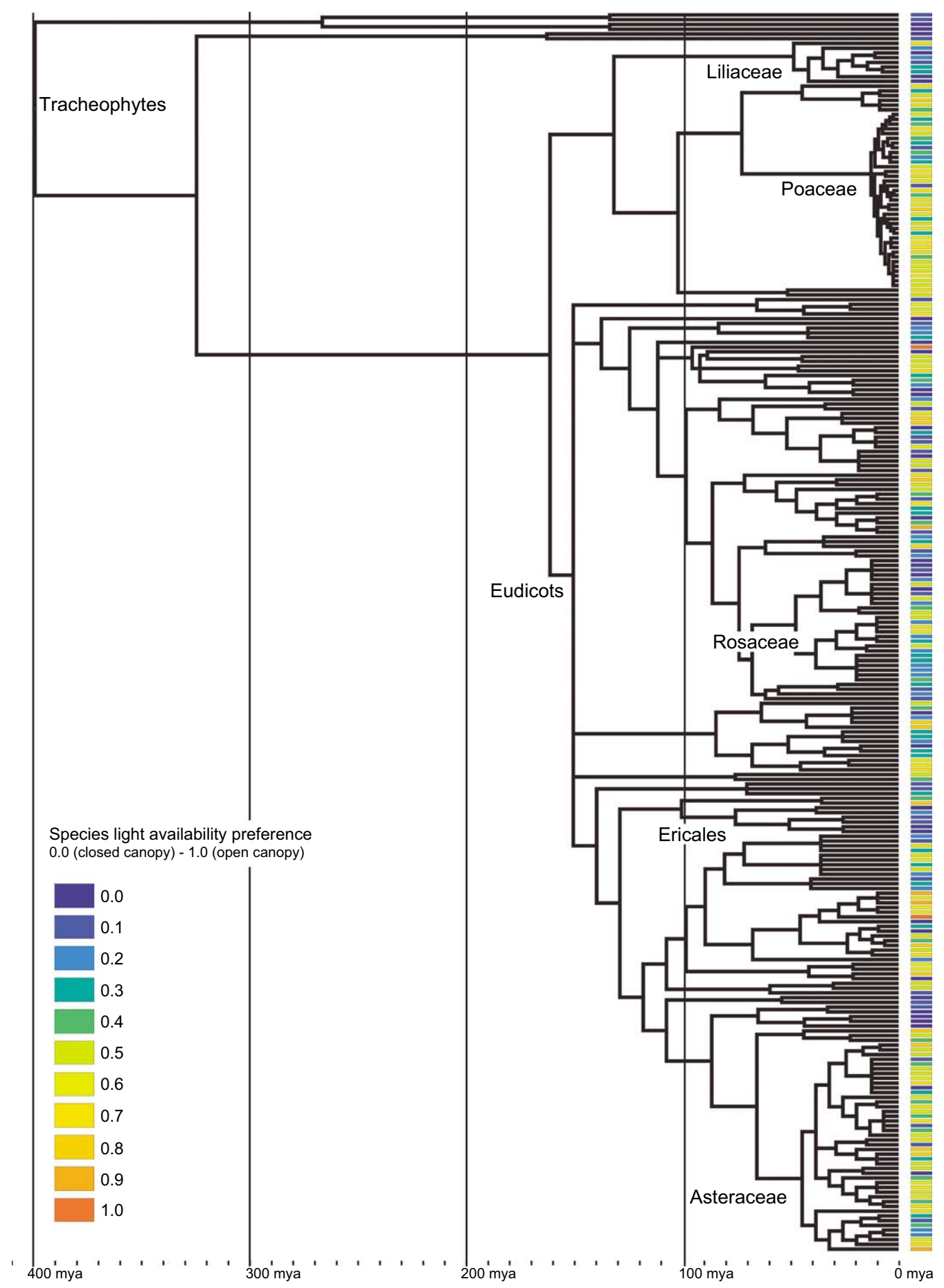

Figure 2. Phylogeny of Cedar Creek oak savanna community $(n=261)$. Colors on tips of phylogeny indicate species light availability niche: orange $(1)=$ fully open canopy, dark-blue $(0)=$ fully closed canopy. On the bottom is the age of the tree in millions of years based on fossil node estimates. A subset of nodes are labeled for reference. Taxon names are not shown for simplicity, but a complete species list is provided in the online phylogeny (Supplementary material Appendix 2).

expected by chance at the landscape scale (ranking of observed LMS correlation co-efficient, 50th quantile estimate, and 75th quantile estimate: 999/1000, 999/1000, and 999/1000, respectively), indicating phylogenetic clustering. These results were robust to phylogenetic topology, but not branch lengths (Supplementary material Appendix 4).
At the local scale (i.e. neighborhoods within plots) patterns of phylogenetic community structure were highly variable across plots. Plots ranged from significantly clustered to significantly overdispersed; however, the majority of plots were not significantly phylogenetically structured (Supplementary material Appendix 5). In general these results were 
robust to both topology and branch lengths (Supplementary material Appendix 5).

\section{Increasing phylogenetic clustering with environmental variation at constant spatial scale}

As environmental variation within a plot increased, species co-occurring in neighborhoods tended to be more phylogenetically closely related (clustered). Overall, there was a significant correlation between plot phylogenetic structure and within-plot variation in light availability (Fig. 3). Phylogenetic clustering tended to increase with environmental variation. This correlation was robust to topology, branch lengths, and correlation methods (Supplementary material Appendix S6).

There was a significant quadratic correlation between the variance in light availability and its mean $\left(\mathrm{x}^{2}=-0.23, \mathrm{x}=\right.$ $\left.-0.24, \mathrm{R}^{2}=0.50, \mathrm{p}<.001\right)$. This is because light availability is more uniform at both ends of the light environment spectrum (i.e. closed forest and open grasslands plots) and more variable in the intermediate environments (savannah plots). Similarly, there was a significant quadratic relationship between phylogenetic community structure and mean light availability (results not shown). However, when phylogenetic community structure was tested against light availability variance, mean and mean $^{2}$ in the same

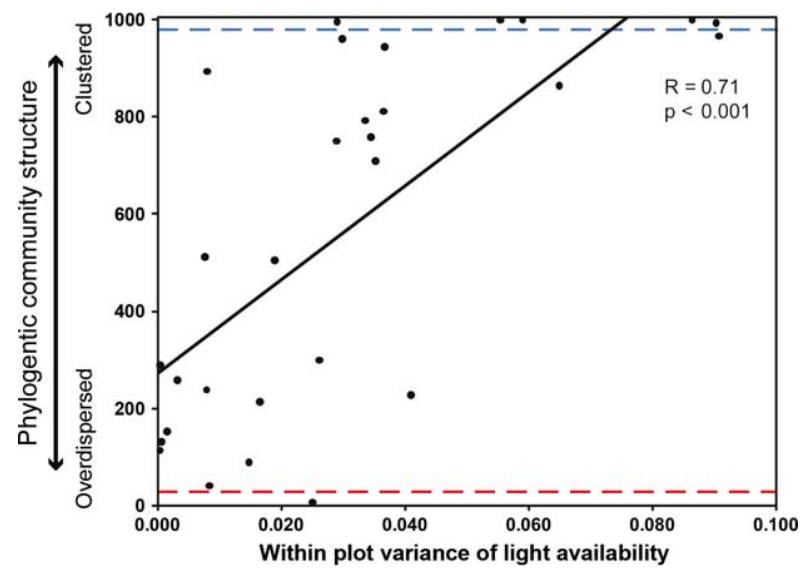

Figure 3. Correlation between community phylogenetic structure and environmental variation with spatial extent held constant. The $\mathrm{x}$-axis represents environmental variation within a plot measured as the variance $\left(\sigma^{2}\right)$ of light availability across neighborhoods; higher variance indicates a steeper light gradient within the plot. The $y$-axis represents phylogenetic community structure measured as the regression slope (75th quantile) between species pairwise cooccurrence in neighborhoods $\left(0.5 \mathrm{~m}^{2}\right)$ vs species pairwise phylogenetic distance. To determine the significance of the observed slope, the observed value is ranked against a null distribution generated by randomizing species occurrences across neighborhoods within each plot ( 0.375 ha) 999 times. If the observed value ranks greater or equal to 975 , the plot is significantly phylogenetically clustered (blue dashed line). If the observed value ranks less or equal to 25 , the plot is significantly phylogenetically overdispersed (red dashed line). Each circle represents one plot $(n=29)$. The correlation was similar when phylogenetic community structure was determined using LMS correlation (Results, Supplementary material Appendix 6). generalized linear model, phylogenetic community structure was significantly correlated with the variance of light availability $(\beta=8265, \mathrm{t}=2.96, \mathrm{p}=0.007)$, but not the mean or mean ${ }^{2}$ light availability $(\beta=842, \mathrm{t}=0.86, \mathrm{p}=$ 0.398 and $\beta=-938, \mathrm{t}=-0.94, \mathrm{p}=0.357$, respectively). Thus, while the variance and mean of light availability covary to certain a degree, phylogenetic community structure is best predicted by the variance rather than by the mean.

\section{Discussion}

The phylogenetic community structure of Minnesota oak savanna understory varied with spatial extent. Communities were phylogenetically clustered at the landscape scale $(\sim 1000 \mathrm{ha})$, while, at the local scale $(0.375 \mathrm{ha})$ phylogenetic community structure varied between clustering and overdispersion. Furthermore, we found: 1) significant associations between species functional traits and species light availability niches, and 2) phylogenetic signal for both species functional traits and light availability niches. Given that the functional traits observed reflect well characterized ecological strategies with specific costs and benefits under differing environmental conditions, these results suggest that species are sorting along a light availability gradient based on phylogenetically conserved functional traits, likely explaining the patterns of phylogenetic clustering we observe. Finally, we found that phylogenetic clustering at the local scale increased with environmental variation within plots, as predicted (Fig. 3). Thus, it appears that the importance of environmental filtering of species based on their conserved functional traits is not dependent on spatial scale per se, but on the degree of environmental variation present at any given scale. This lends support to the assumption that the commonly found pattern of increasing ecological or phylogenetic similarity in species with increasing spatial extent can be explained by increasing environmental variation at larger scales. In the following discussion we highlight the general relevance of these findings and discuss mechanisms that might be responsible for the observed patterns.

\section{Assembly processes and structural patterns of Minnesota oak savanna}

Patterns of phylogenetic community structure are thought to result from underlying community assembly processes (Webb et al. 2002), although it is increasingly clear that there is a multiplicity of processes that can cause the same pattern (Cavender-Bares et al. 2009). The pattern of phylogenetic clustering we observe at the landscape scale suggests that environmental filtering is important to the assembly of Minnesota oak savanna communities. However, the interpretation of phylogenetic clustering as evidence of environmental filtering depends on two assumptions: 1) that the biogeography of speciation does not bias the relatedness of species within communities, and 2) that species niches are phylogenetically conserved. In this study, we have addressed both of these potential caveats. 
Firstly, the geography of speciation often results in species within geographic regions being more closely related than across regions (Losos and Glor 2003, Wiens and Donoghue 2004). The geography of speciation can thus give rise to patterns of phylogenetic clustering independent of environmental filtering when communities are compared across broad spatial extents (Cavender-Bares et al. 2009, Vamosi et al. 2009). In our study, which encompassed a relatively small area $(\sim 1000 \mathrm{ha})$, all communities share the same regional species pool, and biogeographic processes are extremely unlikely to differentially influence community structure.

Secondly, the idea that related species share similar ecological niches is long-standing in evolutionary biology (Donoghue 2008). Recent studies have confirmed phylogenetic signal in several plant functional traits (Prinzing et al. 2001, Chave et al. 2006, Kerkhoff et al. 2006, McCarthy et al. 2007, Swenson and Enquist 2007), including SLA (Ackerly and Reich 1999, Ackerly 2004b, Cavender-Bares et al. 2006). Yet, demonstrating a phylogenetic signal in a functional trait is not itself demonstration of niche conservatism (Losos 2008). Indeed, few studies have directly demonstrated niche conservatism in plants (but see Prinzing et al. 2001). Here, we both explicitly demonstrate phylogenetic signal in traits correlated with species light availability preference and in species light availability niche itself. Thus, this study provides a direct link between the phylogenetic patterns of species niches and species functional traits.

The significant correlations between functional traits and species light availability niches (Table 1 ) provide evidence that these traits are functionally important in determining species distribution across a light gradient. This mechanistic explanation is strongly supported by the fact that our results confirm our a priori predictions about the functional relationship of these traits with the light environment (Methods: Trait data). Furthermore, our results add to the growing body of literature on the general importance of functional traits as part of successful ecological strategies that determine species distributions (Weiher et al. 1998, Reich et al. 1999, 2003, Wright et al. 2001, Ackerly 2004a, Cavender-Bares et al. 2004a, McGill et al. 2006, Kraft et al. 2008). Interestingly, SLA and PA remained the only significant variables associated with species light availability niche when traits were tested in a multivariate regression model. While functional importance can be applied to all of the traits we measured, it appears that those functions and collective ecological strategies best approximated by SLA and PA (e.g. the overall position of each species on the leaf economics spectrum) are the most important in determining species distribution across a light gradient.

Moreover, SLA and PA were found to show significant phylogenetic signal. Thus, the sorting of species based on SLA and PA (and the overall set of coordinated leaf economic traits they represent) is likely what underlies the observed phylogenetic signal in species light availability niches. In turn, the phylogenetic signal in both SLA, PA and light availability niche likely underlie the patterns of phylogenetic clustering we observe.

Our results indicate that closely related species that share similar niche preferences tend to co-occur. There are several possible and mutually compatible explanations for how ecologically similar species are able to co-exist. For instance, given sufficient environmental (i.e. niche) variation at every point in time, species that are closely related, but that occupy slightly different positions along an environmental continuum might be successful, given the evidence of success for species from across the leaf economics spectrum in most communities (Reich et al. 1999, Wright et al. 2004). Moreover, biotic interactions between closely related species might be mediated by character displacement along unmeasured niche axes that vary within a given light habitat ( $\alpha$-niche, Silvertown et al. 2006, Prinzing et al. 2008). Because we are comparing species across the entire spectrum of vascular plants, it is reasonable to imagine that they have diverged along abiotic and biotic environmental axes other than light. For example, closely related species may be differentially susceptible to a given set of herbivores and pathogens, permitting co-existence. Alternatively, species may partition themselves along novel temporal niches (Kelly and Bowler 2005). For example, Cavender-Bares et al. $(2004 \mathrm{a}, \mathrm{b})$ suggested a process of temporal niche partitioning to explain the co-existence of red and white oaks through alternating masting years. A study of the community structure of a single clade might reveal a much stronger signal of overdispersion resulting from finer scale niche partitioning among an otherwise ecologically similar group of closely related species, similar to the findings of Cavender-Bares et al. (2004a, b) and Slingsby and Verboom (2006). However, we found no evidence for overdispersion within narrowly defined lineages (Poaceae) in this system (data not shown). Finally, theoretical models predict that competitive interactions themselves could lead to the coevolution of ecologically similar species occurring together across niche axes (Scheffer and van Nes 2006). Regardless of the mechanisms that allow species to co-exist in this system, the interaction between species traits and the environment are likely important in determining their distribution and the subsequent assemblages they compose.

\section{Patterns of phylogenetic community structure and environmental variation}

As noted above, previous studies have found important relationships between spatial scale and community structure such that communities tend to become phylogenetically clustered with increased spatial extent (Cavender-Bares et al. 2006, Slingsby and Verboom 2006, Swenson et al. 2007). One hypothesis to explain this observation is that environmental variation increases between communities across greater spatial extents. This process is similar to the explanation for the predicted increase in $\beta$-diversity with increasing spatial extent (Crist et al. 2003). Simply put, the steeper the environmental gradient, the greater the species turn-over as different species will be better adapted to different environments. In other words, the steeper the environmental gradient the more that environmental filtering will sort species based on their functional traits and niche requirements. If these functional traits and niches are conserved, then phylogenetic clustering will also increase with greater environmental variation (Cavender-Bares et al. 2006, Swenson et al. 2007). 
Here we demonstrated explicitly that phylogenetic clustering increased with environmental variation independent of spatial extent (Fig. 3). As within plot variation in light availability increased, so did the tendency for close relatives to co-occur in small neighborhoods $\left(0.5 \mathrm{~m}^{2}\right)$ within the plot. Thus, even at local spatial scales (0.375 ha), environmental filtering can be an important factor in structuring communities if the environmental gradient is sufficiently steep.

These results provide continued support for the importance of niche conservatism and environmental filtering in community assembly (Ackerly 2004b, Cavender-Bares et al. 2006). Moreover, we provide a plausible mechanistic explanation for the patterns we observed based on the interplay between a common environmental gradient, light availability, and the evolutionary history of two important functional traits, SLA and PA. In particular, the importance of SLA and light availability in determining species distribution in numerous vascular plant communities is well documented (Kitajima 1994, Wright et al. 2001, Reich et al. 2003, Ackerly 2004a, b, Peterson and Reich 2008). Furthermore, SLA has been found to be widely phylogenetically conserved (Ackerly and Reich 1999, Ackerly 2004b, Cavender-Bares et al. 2006). The general functional importance and phylogenetically conservatism of SLA and, at least potentially, PA could have broad implications for how species respond to changing environments. If, for instance, these conserved traits are relevant to changing conditions then species' responses might be biased toward migration rather than adaptation (Donoghue 2008). This has been shown to be the case in the Californian chaparral. Its modern composition appears to be the result of historic migration of species into the novel chaparral habitat and subsequent environmental sorting based on functional traits evolved ex situ (Ackerly 2004b). For this reason, predictions about species and ecosystem response to future climate change might be better informed by incorporating the shared evolutionary history of relevant functional traits into niche modeling exercises (Edwards et al. 2007).

\section{Conclusion}

The understory vascular plant communities of Minnesota oak savanna are sorting along the environmental gradient of light availability based on species functional traits and their light availability niches. Both functional traits and light availability niches are phylogenetically conserved. Hence, we conclude that environmental filtering has caused phylogenetic clustering at both broad $(\sim 1000$ ha $)$ and local $(0.375 \mathrm{ha})$ spatial scales where there was sufficient environmental variation to influence the distribution of species based on their functional traits. Our results suggest that the extent of environmental variation rather than spatial extent alone should be accounted for when interpreting patterns of phylogenetic community structure, and that environmental filtering, particularly along light gradients, is a critical mechanism in vascular plant community assembly.

Acknowledgements - All authors contributed intellectual input to the project. CGW and JCB wrote the paper. JCB and CGW designed the study. CGW collected the trait data and analyzed the data with $\mathrm{MH}$ and SM. JCB and CL wrote the programs for the community phylogenetic analyses with AK. AK and CGW developed the phylogeny with JCB. PBR contributed all of the community and environmental data and analysis of intraspecifc variation in specific leaf area. We thank Jared Trost and the many summer interns for assistance with the field work at Cedar Creek. We thank Olivier Hardy for testing the statistical performance of our null models. We thank Zhenxiang Xi for help with analysis scripting. We are also grateful to David Ackerly, Steven Kembel, Jonathan Losos, Nathan Swenson, Jens-Christian Svenning, Catherine Rushworth, and two anonymous referees for thoughts and comments on the manuscript. Financial support was provided by the Long-Term Ecological Research program of the National Science Foundation to JCB and PBR [DEB-0620652] and an Undergraduate Research Opportunity (UROP) grant from the Univ. of Minnesota to CGW. We also thank David Tilman for his contribution to an earlier phase of this project.

\section{References}

Ackerly, D. D. 2004a. Functional traits of chaparral shrubs in relation to seasonal water deficit and disturbance. - Ecol. Monogr. 74: 25-44.

Ackerly, D. D. 2004b. Adaptation, niche conservatism, and convergence: comparative studies of leaf evolution in the California chaparral. - Am. Nat. 163: 654-671.

Ackerly, D. D. and Reich, P. B. 1999. Convergence and correlations among leaf size and function in seed plants: a comparative test using independent contrasts. - Am. J. Bot. 86: $1272-1281$.

Allen, T. F. H. and Starr, T. B. 1982. Hierarchy: perspectives for ecological complexity. - Univ. of Chicago Press.

Bazzaz, F. A. 1996. Plants in changing environments: linking physiological, population, and community ecology. - Cambridge Univ. Press.

Cavender-Bares, J. and Lehman, C. 2007. EcoPhyl. - <www.cbs. umn.edu/cavender/ecophylogenetics.shtml $>$.

Cavender-Bares, J. et al. 2004a. Multiple trait associations in relation to habitat differentiation among 17 Florida oak species. - Ecol. Monogr. 74: 635-662.

Cavender-Bares, J. et al. 2004b. Phylogenetic overdispersion in Floridian oak communities. - Am. Nat. 163: 823-843.

Cavender-Bares, J. et al. 2006. Phylogenetic structure of Floridian plant communities depends on taxonomic and spatial scale. - Ecology 87: S109-S122.

Cavender-Bares, J. et al. 2009. The merging of community ecology and phylogenetic biology. - Ecol. Lett. 12: 693-715.

Chase, J. M. and Leibold, M. A. 2003. Ecological niches: linking classical and contemporary approaches. - Univ. of Chicago Press.

Chave, J. et al. 2006. Regional and phylogenetic variation of wood density across 2456 neotropical tree species. - Ecol. Appl. 16: 2356-2367.

Chesson, P. L. 1991. A need for niches? - Trends Ecol. Evol. 6: 26-28.

Crist, T. O. et al. 2003. Partitioning species diversity across landscapes and regions: a hierarchical analysis of alpha, beta, and gamma diversity. - Am. Nat. 162: 734-743.

Darwin, C. 1859. On the origin of species. - John Murray.

Diamond, J. M. 1975. Assembly of species communities. - In: Cody, M. L. and Diamond, J. M. (eds), Ecology and evolution of communities. Harvard Univ. Press, pp. 342-444.

Donoghue, M. J. 2008. A phylogenetic perspective on the distribution of plant diversity. - Proc. Nat. Acad. Sci. USA 105: 11549-11555. 
Dybzinski, R. and Tilman, D. 2007. Resource use patterns predict long-term outcomes of plant competition for nutrients and light. - Am. Nat. 170: 305-318.

Edwards, E. J. et al. 2007. The relevance of phylogeny to studies of global change. - Trends Ecol. Evol. 22: 243-249.

Emerson, B. C. and Gillespie, R. G. 2008. Phylogenetic analysis of community assembly and structure over space and time. - Trends Ecol. Evol. 23: 619-630.

Fargione, J. et al. 2003. Community assembly and invasion: an experimental test of neutral versus niche processes. - Proc. Nat. Acad. Sci. USA 100: 8916-8920.

Felsenstein, J. 1985. Phylogenies and the comparative method. - Am. Nat. 125: 1-15.

Givnish, T. J. and Vermeij, G. J. 1976. Sizes and shapes of liane leaves. - Am. Nat. 110: 743-778.

Hardy, O. J. 2008. Testing the spatial phylogenetic structure of local communities: statistical performances of different null models and test statistics on a locally neutral community. - J. Ecol. 96: 914-926.

Hardy, O. J. and Senterre, B. 2007. Characterizing the phylogenetic structure of communities by an additive partitioning of phylogenetic diversity. - J. Ecol. 95: 493-506.

Hubbell, S. P. 1979. Tree dispersion, abundance and diversity in a tropical dry forest. - Science 203: 1299-1309.

Hubbell, S. P. 2001. The unified neutral theory of biodiversity and biogeography. - Princeton Univ. Press.

Kelly, C. K. and Bowler, M. G. 2005. A new application of storage dynamics: differential sensitivity, diffuse competition, and temporal niches. - Ecology 86: 1012-1022.

Kembel, S. and Hubbell, S. P. 2006. The phylogenetic structure of a neotropical forest tree community. - Ecology 87: 86-99.

Kembel, S. W. et al. 2008. Picante: phylocom integration, community analyses, null-models, traits and evolution in $\mathrm{R}$ v. 0.2-0. - <http://picante.r-forge.r-project.org/>.

Kerkhoff, A. J. et al. 2006. Phylogenetic and growth form variation in the scaling of nitrogen and phosphorus in the seed plants. - Am. Nat. 168: E103-E122.

King, D. A. 1990. The adaptive significance of tree height. - Am. Nat. 135: 809-828.

Kitajima, K. 1994. Relative importance of photosynthetic traits and allocation patterns as correlates of seedling shade tolerance of 13 tropical trees. - Oecologia 98: 419-428.

Kozak, K. H. et al. 2005. Phylogenetic analysis of ecomorphological divergence, community structure, and diversification rates in dusky salamanders (Plethodontidae: Desmognathus). - Evolution 59: 2000-2016.

Kozlowski, T. T. and Pallardy, S. G. 2002. Acclimation and adaptive responses of woody plants to environmental stresses. - Bot. Rev. 68: 270-334.

Kraft, N. J. B. et al. 2008. Functional traits and niche-based tree community assembly in an amazonian forest. - Science 322: 580-582.

Legendre, P. et al. 2002. The consequences of spatial structure for the design and analysis of ecological field surveys. - Ecography 25: 601-615.

Leibold, M. and McPeek, M. A. 2006. Coexistence of the niche and neutral perspectives in community ecology. - Ecology 87 : $1399-1410$.

Losos, J. B. 2008. Phylogenetic niche conservatism, phylogenetic signal and the relationship between phylogenetic relatedness and ecological similarity among species. - Ecol. Lett. 11: 995-1007.

Losos, J. B. and Glor, R. E. 2003. Phylogenetic comparative methods and the geography of speciation. - Trends Ecol. Evol. 18: $220-227$.

Lusk, C. H. and Warton, D. I. 2007. Global meta-analysis shows that relationships of leaf mass per area with species shade tolerance depend on leaf habit and ontogeny. - New Phytol. 176: 764-774.

Machado, J. L. and Reich, P. B. 1999. Evaluation of several measures of canopy openness as predictors of photosynthetic photon flux density in deeply shaded conifer-dominated forest understory. - Can. J. For. Res. 29: 1438-1444.

Maddison, W. P. and Maddison, D. R. 2006. Mesquite: a modular system for evolutionary analysis. - Mesquite. v. 2.6, $<$ www.mesquiteproject.org $>$.

McCarthy, M. C. et al. 2007. Organ partitioning and distribution across the seed plants: assessing the relative importance of phylogeny and function. - Int. J. Plant Sci. 168: 751-761.

McGill, B. J. et al. 2006. Rebuilding community ecology from functional traits. - Trends Ecol. Evol. 21: 178-185.

Menges, E. S. and Kohfeldt, N. 1995. Life history strategies of Florida scrub plants in relation to fire. - Bull. Torrey Bot. Club 122: 282-297.

Paradis, E. and Claude, J. 2002. Analysis of comparative data using generalized estimating equations. $-\mathrm{J}$. Theor. Biol. 218: $175-185$.

Paradis, E. et al. 2004. APE: analyses of phylogenetics and evolution in R language. - Bioinformatics 20: 289-290.

Parrish, J. A. D. and Bazzaz, F. A. 1976. Niche separation in roots of successional plants. - Ecology 57: 1281-1288.

Peterson, D. W. and Reich, P. B. 2008. Fire frequency and tree canopy structure influence plant species diversity in a forestgrassland ecotone. - Plant Ecol. 194: 5-16.

Prinzing, A. et al. 2001. The niche of higher plants: evidence for phylogenetic conservatism. - Proc. R. Soc. B 268: 2383-2389.

Prinzing, A. et al. 2008. Less lineages - more trait variation: phylogenetically clustered plant communities are functionally more diverse. - Ecol. Lett. 11: 809-819.

Rasband, W. S. 1997-2006. ImageJ v. 1.36. - National Inst. of Health.

Reich, P. B. et al. 1999. Generality of leaf trait relationships: a test across six biomes. - Ecology 80: 1955-1969.

Reich, P. B. et al. 2003. The evolution of plant functional variation: traits, spectra, and strategies. - Int. J. Plant Sci. 164: S143-S164.

Ricklefs, R. E. 1987. Community diversity: relative roles of local and regional processes. - Science 235: 167-171.

Ricklefs, R. E. and Schluter, D. 1993. Species diversity: an introduction to the problem. - In: Ricklefs, R. E. and Schluter, D. (eds), Species diversity in ecological communities: historical and geographical perspectives. Univ. of Chicago Press, pp. 1-10.

Ricklefs, R. E. et al. 2004. The region effect on mesoscale plant species richness between eastern Asia and eastern North America. - Ecography 27: 129-136.

Sack, L. et al. 2003. The 'hydrology' of leaves: co-ordination of structure and function in temperate woody species. - Plant Cell Environ. 26: 1343-1356.

Scharf, F. S. et al. 1998. Inferring ecological relationships from the edges of scatter diagrams: comparison of regression techniques. - Ecology 79: 448-460.

Scheffer, M. and van Nes, E. H. 2006. Self-organized similarity, the evolutionary emergence of groups of similar species. - Proc. Nat. Acad. Sci. USA 103: 6230-6235.

Schluter, D. 2000. The ecology of adaptive radiation. - Oxford Univ. Press.

Schoener, T. W. 1970. Nonsychronous spatial overlap of lizards in patchy habitats. - Ecology 51: 408-418.

Silvertown, J. et al. 1999. Hydrologically defined niches reveal a basis for species richness in plant communities. - Nature 400: 61-63.

Silvertown, J. et al. 2006. Absence of phylogenetic signal in the niche structure of meadow plant communities. - Proc. R. Soc. B 273: 39-44. 
Slingsby, J. A. and Verboom, G. A. 2006. Phylogenetic relatedness limits co-occurrence at fine spatial scales: evidence from the Schoenoid sedges (Cyperaceae: Schoeneae) of the Cape Floristic Region, South Africa. - Am. Nat. 168: 14-27.

Swenson, N. G. and Enquist, B. J. 2007. Ecological and evolutionary determinants of a key plant functional trait: wood density and its community-wide variation across latitude and elevation. - Am. J. Bot. 94: 451-459.

Swenson, N. G. et al. 2007. The influence of spatial and size scale on phylogenetic relatedness in tropical forest communities. - Ecology 88: 1770-1780.

Tilman, D. 1982. Resource competition and community structure. - Princeton Univ. Press.

Tilman, D. 2004. Niche tradeoffs, neutrality, and community structure: a stochastic theory of resource competition, invasion, and community assembly. - Proc. Nat. Acad. Sci. USA 101: 10854-10861.

USDA NRCS PLANTS Database 2006. - < http://plants.usda. gov/>.

Vamosi, S. M. et al. 2009. Emerging patterns in the comparative analysis of phylogenetic community structure. - Mol. Ecol. 18: 572-592.

Wainwright, P. C. et al. 2005. Many-to-one mapping of form to function: a general principle in organismal design? - Integr. Comp. Biol. 45: 256-262.

Webb, C. O. 2000. Exploring the phylogenetic structure of ecological communities: an example for rain forest trees. $-\mathrm{Am}$. Nat. 156: 145-155.

Download the Supplementary material as file E5975 from $<$ www.oikos.ekol.lu.se/appendix $>$.
Webb, C. O. and Donoghue, M. J. 2005. Phylomatic: tree assembly for applied phylogenetics. - Mol. Ecol. Not. 5: $181-183$.

Webb, C. O. et al. 2002. Phylogenies and community ecology. - Annu. Rev. Ecol. Syst. 33: 475-505.

Webb, C. O. et al. 2008. Phylocom: software for the analysis of phylogenetic community structure and trait evolution. - Phylocom v. 3.4, <www.phylodiversity.net/phylocom/>.

Weiher, E. and Keddy, P. 1999. Ecological assembly rules: perspectives, advances, retreats. - Cambridge Univ. Press.

Weiher, E. et al. 1998. Community assembly rules, morphological dispersion, and the coexistence of plant species. - Oikos 81: 309-322.

Wiens, J. and Graham, C. 2005. Niche conservatism: integrating evolution, ecology, and conservation biology. - Annu. Rev. Ecol. Syst. 36: 519-539.

Wiens, J. J. and Donoghue, M. J. 2004. Historical biogeography, ecology and species richness. - Trends Ecol. Evol. 19: 639-644.

Wikström, N. et al. 2001. Evolution of the angiosperms: calibrating the family tree. - Proc. R. Soc. B 268: 2211-2220.

Wright, I. J. et al. 2001. Strategy shifts in leaf physiology, structure and nutrient content between species of high- and low-rainfall and high- and low-nutrient habitats. - Funct. Ecol. 15: 423-434.

Wright, I. J. et al. 2004. The worldwide leaf economics spectrum. - Nature 428: 821-827. 